International Journal of Pure and Applied Mathematics

Volume 106 No. 1 2016, 317-331

ISSN: 1311-8080 (printed version); ISSN: 1314-3395 (on-line version)

url: http://www.ijpam.eu

doi: 10.12732/ijpam.v106i1.26

\title{
ANALYSIS OF ECOLOGICAL MODEL WITH HOLLING TYPE IV FUNCTIONAL RESPONSE
}

\author{
Safaa Jawad Ali ${ }^{1,2}$, Norihan Md. Arifin ${ }^{1}$, Raid Kamel Naji ${ }^{3}$, \\ Fudziah Ismail ${ }^{1}$, Norfifah Bachok ${ }^{1}$ \\ ${ }^{1}$ Institute for Mathematical Research \\ University Putra Malaysia \\ MALAYSIA \\ ${ }^{2}$ Technical Instructor Preparing Institute \\ Middle Technical University \\ Baghdad, IRAQ \\ ${ }^{3}$ Department of Mathematics \\ College of Science \\ University of Baghdad \\ IRAQ
}

\begin{abstract}
In this paper a three-species of predator-prey food chain model with Holling type IV functional response is proposed. The boundedness of the solution of the model is discussed. Stability analysis of the system is carried out. The dynamics of the predator-prey food chain model with simplified Holling type IV functional response is investigated theoretically as well as numerically.
\end{abstract}

AMS Subject Classification: 37D45

Key Words: food chain, bifurcation, Holling Type IV, periodic, chaotic

\section{Introduction}

Ecological models of intracting population dynamics in nature, complex and non-linear. Many researchers have been reported the complex dynamics of a

Received: November 19, 2015

Published: February 5, 2016

${ }^{\S}$ Correspondence author
(C) 2016 Academic Publications, Ltd.

url: www.acadpubl.eu 
predator-prey food chains and food web, see [8],[9],[13] and [15]. May [17] proposed chaos and chaotic dynamics in a simple model which is well known by the logistic map and brought out unpredictable dynamics of chaotic. Rai [19] proposed a three species food chain model and explained the presence of a period-doubling scenario leading to chaos. Still the work of Hasting and Powell [13] among others [4-9],[12-16] and [18] is the most cited work. In their article, the model was first formulated, stability analysis and Hopf bifurcation were performed. An important factor discovered by Abrams [1], Abrams and Roth [2], and futher discussed in De Feo and Rinaldi [7] is that the main value of density of the top population is maximum at the transition from cyclic to chaotic regimes. Haque [12] studied the impact of intraspecific competition of tri-trophic food chain model, theoretical and numerical results shows that intraspecific competition plays an important role in controlling the chaotic dynamics of the system. This will confirmed in this article.

A suitable mathematical model in ecology and biology cannot easily achieved because this model could never predict nature's behavior. To face this major problem we can search for analyzable model that can be close enough the reality. Various mathematical tools, like numerical simulation and bifurcation diagrams have been used to detect chaotic dynamics in predator-prey of multi species ecological models. The functional response defined by Lotka in 1925 and Volterra in 1926 for a predator-prey model is linear and unbounded, while studying the complexity in model ecosystems need reasonable functional responces that should be nonlinear and bounded, see [17] and [22]. In 1959 Holling [14] used Michaelis-Menten's formula $\frac{w x}{c+x}$ as one of predator functional response. It is know well-known as a Holling type II or Michaelis-Menten functional response. Androws [3] proposed a response $\frac{w x}{c+d x+x^{2}}$ called the Monod-Haldane functional response. Collings [6] used this function and called it Holling type IV response. This response function describes a sittuation in which the predator's per capita rate of predation decreases at sufficiently high prey densities [20]. In their experiments about the kinetics of phenol oxidation Sokol and Howell [23] suggested a simplified Holling type IV function of the form $\frac{w x}{d+x^{2}}$ and found that it is simpler and better than the original function of Holling type IV. Many investigations with Holling type II functional response, while few investigations using Holling type IV functional response in population ecology [20-23].

This paper is organized as follows: In Section 2, the mathematical model is proposed and each parameter in the model is described. In Section 3, the boundedness of the solution of the model is established. Stability analysis of the equilibrium points of the model are derived in Section 4. In Section 5, numerical study are carried out to obtain the behavior of the model. Finally, 
the paper is end with a discussion and results in Section 6 .

\section{The Mathematical Model}

Consider the three species food chain model at time $t$ consisting of the prey whose population density denoted by $x(t)$, the intermediate predator whose population density denoted by $y(t)$ and the top predator whose population density denoted by $z(t)$. The intermediate predator $y$ preys on its sole food $x$ at the lower level according to the simplified Holling type-IV functional response, while the top predator $z$ preys on $y$ at the second level according to Holling Type II functional response.

The dynamics of the above food chain model can be represented by the following set of differential equations:

$$
\begin{aligned}
\frac{d x}{d t} & =x\left(a_{1}-b_{1} x\right)-\frac{m_{0} x y}{h_{1}+x^{2}}: x(0) \geq(0), \\
\frac{d y}{d t} & =\frac{m_{1} x y}{h_{1}+x^{2}}-d_{1} y-\frac{m_{2} y z}{h_{2}+y}: y(0) \geq(0), \\
\frac{d z}{d t} & =\frac{m_{3} y z}{h_{2}+y}-d_{2} z: z(0) \geq 0 .
\end{aligned}
$$

Here the positive constants $a_{1}, b_{1}, h_{i}, d_{i}(i=1,2)$ and $m_{j}(j=0,1.2,3)$, denote to: $a_{1}$ is the growth rate of the prey $x, b_{1}$ represents the intraspecific competition among individuals of prey $x, h_{i}{ }^{\prime} s$ are the half-saturation constant, $d_{i}{ }^{\prime} s$ are the predator's death rate, $m_{0}$ is the maximum value which per capita reduction rate can $\mathrm{x}$ attain, $m_{1}$ has similar meaning to $m_{0}, m_{2}$ is the maximum value which per capita reduction rate can y attain, $m_{3}$ has similar meaning to $m_{2}$. Then the system of the three species food chain model given by (1) can be written as follows:

$$
\begin{aligned}
\frac{d x}{d t} & =x\left(a_{1}-b_{1} x-\frac{m_{0} y}{h_{1}+x^{2}}\right)=G_{1}(x, y, z) \\
\frac{d y}{d t} & =y\left(\frac{m_{1} x}{h_{1}+x^{2}}-d_{1}-\frac{m_{2} z}{h_{2}+y}\right)=G_{2}(x, y, z) \\
\frac{d z}{d t} & =z\left(\frac{m_{3} y}{h_{2}+y}-d_{2}\right)=G_{3}(x, y, z)
\end{aligned}
$$

with $x(0) \geq 0, y(0) \geq 0, z(0) \geq 0$. Obviously the interaction functions $G_{i}$ $(i=1,2,3)$ of system $(2)$ are continuous and have continuous partial derivatives 
on the positive octant $R_{+}^{3}=\left\{(x, y, z) \in R_{+}^{3}: x \geq 0, y \geq 0, z \geq 0\right\}$. Therefore these functions are Lipschitzian on $R_{+}^{3}$, and hence the solution of the system (2) exists and is unique.

\section{Boundedness of the Model}

In this section, the boundedness of the solution of the system (2) in $R_{+}^{3}$ is established, as shown in the following theorem.

Theorem 1. All the solutions of the three species food chain system (2) are uniformly bounded in $R_{+}^{3}$, and ultimately entering the region

$$
\begin{gathered}
\Omega=\left\{(x, y, z) \in R_{+}^{3}: 0 \leq x \leq \frac{a_{1}}{b_{1}}, 0 \leq M_{1} \leq \frac{m_{1} a_{1}}{m_{0} b_{1} d_{1}}\left(a_{1}+d_{1}\right),\right. \\
\left.0 \leq M_{2} \leq \frac{m_{1} a_{1}}{m_{0} b_{1} L}\left(a_{1}+L\right)\right\}
\end{gathered}
$$

where $M_{1}=\frac{m_{1}}{m_{0}} x+y, M_{2}=\frac{m_{1}}{m_{0}} x+y+\frac{m_{2}}{m_{3}} z$ and $L=\min \left\{d_{1}, d_{2}\right\}$.

Proof. Let $(x(t), y(t), z(t))$ be any solution of the system with non-negative initial condition.

From the first equation of system (2) we have

$$
\frac{d x}{d t} \leq x\left(a_{1}-b_{1} x\right)
$$

then according to comparison theorem [11], we obtain that

$$
\lim _{t \rightarrow \infty} \operatorname{Sup} x(t) \leq \frac{a_{1}}{b_{1}}
$$

implies that $x(t) \leq \frac{a_{1}}{b_{1}}, \forall t \geq 0$. Now as $t \rightarrow \infty$.

$$
\Rightarrow 0 \leq x(t) \leq \frac{a_{1}}{b_{1}}
$$

Consider now $M_{2}(t)=\frac{m_{1}}{m_{0}} x+y+\frac{m_{2}}{m_{3}} z$. Then

$$
\begin{aligned}
\frac{d M_{2}(t)}{d t} & =\frac{m_{1}}{m_{0}} a_{1} x-b_{1} x^{2}-d_{1} y-\frac{m_{2}}{m_{3}} d_{2} z \\
& \leq \frac{m_{1} a_{1}}{m_{0} b_{1}}\left(a_{1}+L\right)-L\left(\frac{m_{1}}{m_{0}} x+y+\frac{m_{2}}{m_{3}} z\right)
\end{aligned}
$$


where $L=\min \left\{d_{1}, d_{2}\right\}$. Accordingly, we obtain the following differential inequality

$$
\frac{d M_{2}(t)}{d t}+L M_{2} \leq \frac{m_{1} a_{1}}{m_{0} b_{1}}\left(a_{1}+L\right) .
$$

Again, by applying the comparison theorem [11] we obtain that for $t \rightarrow \infty$,

$$
0 \leq M_{2} \leq \frac{m_{1} a_{1}}{m_{0} b_{1} L}\left(a_{1}+L\right) \forall t \geq 0
$$

Therefore, every solution initiating in non-negative octant are attracted in a bounded set $\Omega$ defined above (Note that $M_{1}$ is bounded similar to $M_{2}$ ).

\section{Stability of the Model}

In this section, the local stability analysis of the non-negative equilibrium points of system (2) are discussed. There are at most four non-negative equilibrium points of system (2), existence and stability conditions of them are given below:

- The trivial equilibrium point $E_{0}=(0,0,0)$ always exits.

- The equilibrium point $E_{1}=\left(\frac{a_{1}}{b_{1}}, 0,0\right)$ always exists on the boundary of the first octant.

- The intermediate predator can exists and survive depending on its prey. Therefore, the equilibrium point $E_{2}=(\bar{x}, \bar{y}, 0)$ exists uniqly in the positive quadrant of $x-y$ plane, where $\bar{x}$ and $\bar{y}$ are given by:

$$
\bar{x}=\frac{m_{1}}{2 d_{1}}, \quad \bar{y}=\frac{1}{m_{0}}\left(a_{1}-b_{1} \frac{m_{1}}{2 d_{1}}\right)\left(h_{1}+\bar{x}^{2}\right),
$$

provided that the following conditions hold

$$
\frac{m_{1}}{2 d_{1}}<\frac{a_{1}}{b_{1}}, m_{1}^{2}-4 d_{1}^{2} h_{1}=0
$$

- In the absence of prey $x$, then both $y$ and $z$ can not survive, so there is no equilibrium point in the $y-z$ plane. In addition to that, if the intermediate predator $y$ is absence, then there is no equilibrium point in $x-z$ plane. 
- The positive equilibrium point $E_{3}=\left(x^{*}, y^{*}, z^{*}\right)$ exists in the interior of the first octant if and only if there is a positive solution to the following equations:

$$
\begin{aligned}
f_{1} & =a_{1}-b_{1} x-\frac{m_{0} y}{h_{1}+x^{2}}=0, \\
f_{2} & =\frac{m_{1} x}{h_{1}+x^{2}}-d_{1}-\frac{m_{2} z}{h_{2}+y}=0, \\
f_{3} & =\frac{m_{3} y}{h_{2}+y}-d_{2}=0 .
\end{aligned}
$$

Straightforward computation shows that

$$
y^{*}=\frac{d_{2} h_{2}}{m_{3}-d_{2}},
$$

while $x^{*}$ is the positive root of the following equation

$$
x^{3}-\frac{a_{1}}{b_{1}} x^{2}+h_{1} x+\left[\frac{1}{b_{1}}\left(m_{0} y^{*}-a_{1} h_{1}\right)\right]=0,
$$

Now since $0 \leq x^{*} \leq \frac{a_{1}}{b_{1}}$, then $f(0)=\left[\frac{1}{b_{1}}\left(m_{0} y^{*}-a_{1} h_{1}\right)\right]<0$, if

$$
y^{*}<\frac{a_{1} h_{1}}{m_{0}}
$$

Also, $f\left(\frac{a_{1}}{b_{1}}\right)=\frac{m_{0}}{b_{1}} y^{*}>0$. Thus, $f(0) f\left(\frac{a_{1}}{b_{1}}\right)<0$, and then there is a positive root of eq.(10) lies in $\left(0, \frac{a_{1}}{b_{1}}\right)$ provided that condition (11) satisfied.

Further, the second equation of (8) gives

$$
z^{*}=\frac{\left(h_{2}+y^{*}\right)}{m_{2}}\left(\frac{m_{1} x^{*}}{h_{1}+x^{* 2}}-d_{1}\right)
$$

Therefore, the positive equilibrium point $E_{3}=\left(x^{*}, y^{*}, z^{*}\right)$ exists if in addition to condition (11) the following conditions hold:

$$
\begin{aligned}
& m_{3}>d_{2}, \\
& \frac{m_{1} x^{*}}{\left(h_{1}+x^{* 2}\right)}>d_{1} .
\end{aligned}
$$

Now in order to investigate the dynamical behavior of the three species food chain system (2) near the above equilibrium points. The variational matrix $V$ of the system $(2)$ at $(x, y, z)$ is computed as: 


$$
V(x, y, z)=\left[\begin{array}{lcr}
x \frac{\partial f_{1}}{\partial x}+f_{1} & x \frac{\partial f_{1}}{\partial y} & x \frac{\partial f_{1}}{\partial z} \\
y \frac{\partial f_{2}}{\partial x} & y \frac{\partial f_{2}}{\partial y}+f_{2} & y \frac{\partial f_{2}}{\partial z} \\
z \frac{\partial f_{3}}{\partial x} & z \frac{\partial f_{3}}{\partial y} & z \frac{\partial f_{3}}{\partial z}+f_{3}
\end{array}\right],
$$

where

$$
\begin{gathered}
\frac{\partial f_{1}}{\partial x}=-b_{1}+\frac{2 m_{0} x y}{\left(h_{1}+x^{2}\right)}, \quad \frac{\partial f_{1}}{\partial y}=-\frac{m_{0}}{\left(h_{1}+x^{2}\right)}, \quad \frac{\partial f_{1}}{\partial z}=0 \\
\frac{\partial f_{2}}{\partial x}=\frac{m_{1}\left(h_{1}-x^{2}\right)}{\left(h_{1}+x^{2}\right)^{2}}, \quad \frac{\partial f_{2}}{\partial y}=\frac{m_{2} z}{\left(h_{2}+y\right)^{2}}, \quad \frac{\partial f_{2}}{\partial z}=-\frac{m_{2}}{\left(h_{2}+y\right)}, \\
\frac{\partial f_{3}}{\partial x}=0, \frac{\partial f_{3}}{\partial y}=\frac{h_{2} m_{3}}{\left(h_{2}+y\right)^{2}}, \frac{\partial f_{3}}{\partial z}=0 .
\end{gathered}
$$

Further, the stability analysis of the system (2) is carried out and according to the variational matrix $V_{i} ; i=0,1,2,3$ of $E_{i} ; i=0,1,2,3$ respectively the following results are obtained:

$$
\begin{aligned}
V_{0} & =\left[\begin{array}{ccc}
a_{1} & 0 & 0 \\
0 & -d_{1} & 0 \\
0 & 0 & -d_{2}
\end{array}\right], \\
V_{1} & =\left[\begin{array}{ccc}
-a_{1} & -\frac{m_{0} x}{h_{1}+x^{2}} & 0 \\
0 & \frac{m_{1} x}{h_{1}+x^{2}}-d_{1} & 0 \\
0 & 0 & -d_{2}
\end{array}\right], \\
V_{2}= & {\left[\begin{array}{lrr}
\bar{x}\left(-b_{1}+\frac{2 \bar{x}\left(a_{1}-b_{1} \bar{x}\right)}{h_{1}+\bar{x}^{2}}\right) & -\frac{m_{0} \bar{x}}{\left(h_{1}+\bar{x}^{2}\right)} & 0 \\
\frac{m_{1} \bar{y}\left(h_{1}-\bar{x}^{2}\right)}{\left(h_{1}+\bar{x}^{2}\right)^{2}} & \frac{m_{1} \bar{x}}{h_{1}+\bar{x}^{2}}-d_{1} & \frac{-m_{2} \bar{y}}{h_{2}+\bar{y}} \\
0 & 0 & \frac{m_{3} \bar{y}}{h_{2}+\bar{y}}-d_{2}
\end{array}\right] . }
\end{aligned}
$$

- Here the trivial equilibrium point $E_{0}$ is saddle-node, having an unstable manifold along $x$-direction and stable manifold along $x-y$ plane.

- From variational matrix $V_{1}$, it is observed equilibrium point $E_{1}$ is a saddle point having stable manifold along $x$ and $z$-direction if the following condition holds

$$
\frac{m_{1} x}{h_{1}+x^{2}}>d_{1}
$$

while the equilibrium point $E_{1}$ having stable manifold along $x, y$, and $z$-direction if the following condition holds

$$
\frac{m_{1} x}{h_{1}+x^{2}}<d_{1} \text {. }
$$


- The equilibrium point $E_{2}=(\bar{x}, \bar{y}, 0)$ according to the characteristic equation of $V\left(E_{2}\right)$ having stable manifold along $x$ and $y$-direction if the following conditions hold respectively

$$
\begin{aligned}
\frac{2 \bar{x}\left(a_{1}-b_{1} \bar{x}\right)}{h_{1}+\bar{x}^{2}} & <b_{1}, \\
\frac{m_{1} \bar{x}}{h_{1}+\bar{x}^{2}} & <d_{1} .
\end{aligned}
$$

It is easy to verify that the root of this equation satisfy the following relations:

$$
\begin{aligned}
\lambda_{1}+\lambda_{2} & =\frac{\bar{x}\left(m_{1}+2\left(a_{1}-b_{1} \bar{x}\right)\right)-\left(b_{1} \bar{x}+d_{1}\right)\left(h_{1}+\bar{x}^{2}\right)}{h_{1}+\bar{x}^{2}} \\
\lambda_{1} \lambda_{2} & =\frac{2 \bar{x}^{2}\left(a_{1}-b_{1} \bar{x}\right)\left(m_{1} \bar{x}-d_{1}\right)+b_{1} \bar{x}\left(d_{1}\left(h_{1}+\bar{x}^{2}\right)-m_{1} \bar{x}\right)}{h_{1}+\bar{x}^{2}}, \\
\lambda_{3} & =\frac{m_{3} \bar{y}}{h_{2}+\bar{y}}-d_{2} .
\end{aligned}
$$

Notice that $\lambda_{1}+\lambda_{2}<0$ and $\lambda_{1} \lambda_{2}>0$ (i.e condition (17) and (18) hold), hence $E_{2}$ is locally asymptotically stable in the $\operatorname{Int} R_{+}^{2}$ of the $x-y$ plane. In addition, since the eigenvalue $\lambda_{3}$ describes the dynamics in the $z$-direction orthogonal on the $x-y$ plane. Therefore, if $\frac{m_{3} \bar{y}}{h_{2}+\bar{y}}<d_{2}$ holds, then $E_{2}$ is locally asymptotically stable in the $I n t R_{+}^{3}$. Moreover, if $\frac{m_{3} \bar{y}}{h_{2}+\bar{y}}>d_{2}$ holds, then $E_{2}$ is a saddle point in the $\operatorname{Int} R_{+}^{3}$ with locally stable manifold in $x-y$ plane and with locally unstable manifold in $z$-direction.

However, for the positive equilibrium point $E_{3}=\left(x^{*}, y^{*}, z^{*}\right)$ the variational matrix is:

$$
\begin{gathered}
V_{3}=\left[\begin{array}{lrr}
a_{1}-2 b_{1} x^{*}-\frac{m_{0} y^{*}\left(h_{1}-x^{* 2}\right)}{R^{2}} & -\frac{m_{0} x^{*}}{R} & 0 \\
\frac{m_{1} y^{*}\left(h_{1}-x^{* 2}\right)}{R^{2}} & \frac{m_{1} x^{*}}{R}-d_{1}-\frac{h_{2} m_{2} z^{*}}{Q^{2}} & -\frac{m_{2} y^{*}}{Q} \\
0 & \frac{h_{2} m_{3} z^{*}}{Q^{2}} & 0
\end{array}\right] \\
=\left[a_{i j}\right] .
\end{gathered}
$$

Here $R=\left(h_{1}+x^{* 2}\right)$ and $Q=\left(h_{2}+y^{*}\right)$. The characteristic equation of the variational matrix (19) can written as:

$$
\lambda^{3}+A_{1} \lambda^{2}+A_{2} \lambda+A_{3}=0
$$

where

$A_{1}=-\left(a_{11}+a_{22}\right), A_{2}=a_{11} a_{22}-a_{12} a_{21}-a_{23} a_{32}, A_{3}=a_{11} a_{23} a_{32}$. 
According to Routh-Hurwitz criterion, $E_{3}=\left(x^{*}, y^{*}, z^{*}\right)$ is locally asymptotically stable provided $A_{1}>0, A_{3}>0$ and $\Delta=A_{1} A_{2}-A_{3}>0$.

Now straightforward computations show that, $A_{1}>0$ and $A_{3}>0$ if and only if the following conditions are satisfied:

$$
a_{1}<2 b_{1} x+\frac{m_{0} y^{*}\left(h_{1}-x^{* 2}\right)-m_{1} x^{*} R}{R^{2}}+\left(d_{1}+\frac{h_{2} m_{2} z^{*}}{Q^{2}}\right),
$$

with

$$
x^{* 2}<h_{1}, \quad m_{1}<\frac{m_{0} y^{*}\left(h_{1}-x^{* 2}\right)}{x^{*} R} .
$$

In addition to that, since

$$
A_{1} A_{2}-A_{3}=\left(a_{11}+a_{22}\right)\left(a_{12} a_{21}-a_{11} a_{22}\right)+a_{22} a_{23} a_{32}
$$

Hence, the necessary condition for $A_{1} A_{2}-A_{3}>0$ is $\left(a_{12} a_{21}-a_{11} a_{22}\right)<0$ satisfied, provided that the following condition holds

$$
\begin{gathered}
\left(a_{1}-2 b_{1} x^{*}\right)\left[\frac{m_{1} x^{*}}{R}-\left(a_{1}+\frac{h_{2} m_{2} z^{*}}{Q^{2}}\right)\right] \\
+\frac{m_{0} y^{*}\left(h_{1}-x^{* 2}\right)}{R^{2}}\left(d_{1}+\frac{h_{2} m_{2} z^{*}}{Q^{2}}\right)>0 .
\end{gathered}
$$

Therefore, depending on the above analysis, the locally asymptotically stable in $I n t R_{+}^{3}$ of the positive equilibrium point $E_{3}=\left(x^{*}, y^{*}, z^{*}\right)$ is discussed in the following theorem.

Theorem 2. Suppose that positive equilibrium point $E_{3}=\left(x^{*}, y^{*}, z^{*}\right)$ exists in $I n t R_{+}^{3}$, then $E_{3}$ is locally asymptotically stable provided conditions (20-22) hold.

Proof. Follows directly from Routh-Hurwitz criterion [17].

\section{Numerical Simulation}

In this section the global dynamics of system (2) is studied numerically. The food chain system is solved numerically using predictor-corrector method with six order Runge-Kutta method [10]. System (2) run for 40000 time steps and 
the first 25000 time steps are deleted to eliminate the transient effect. For the following set of fixed parameters values

$$
\begin{array}{r}
b_{1}=0.3, m_{0}=0.1, m_{1}=0.27, m_{2}=0.29, m_{3}=0.095 \\
d_{1}=0.017, d_{2}=0.025, h_{1}=1.0, h_{2}=2.0
\end{array}
$$

The attractor of system (2) in 3D and 2D are drawn for the initial condition (1.2, $1.2,1.2)$. The main objective is to explore the possibility of chaotic behavior of system (2) by varying the control parameter and keeping other parameters of (23) fixed, and there are three cases to explain the behavior of the model in addition to bifurcation diagrams.

The bifurcation diagram with respect to sensitive parameter $a_{1}$ is drawn in Fig.1. The cascades of period doubling is observed in Fig. 1 for $a_{1} \in(0.25,0.5)$ and with data in (23) which is the route of chaos in system (2). The complex dynamics of system (2) is easily observed from bifurcation diagrams.

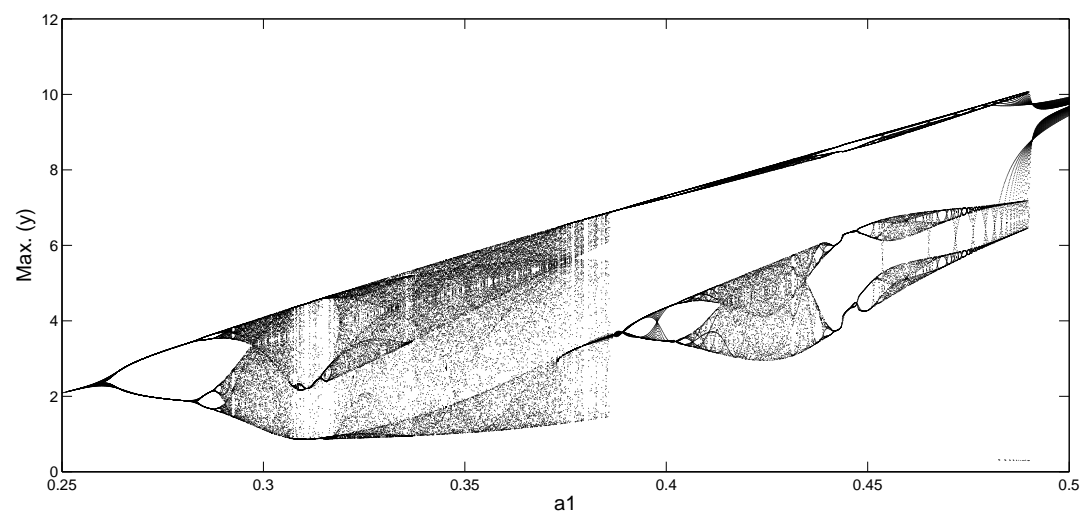

Figure 1: Bifurcation diagram of system (2) for data in (23) the maxima of $y$ as function of $a_{1}$ and $a_{1} \in(0.25,0.5)$.

Now the first case by varying the value of $a_{1}$ in the range $0,35-0.20$, it is observed that the system approaches to chaotic dynamics of system (2) as shown in Fig.2. Decreasing the value of $a_{1}=0.27$ change the behavior of system (2) from chaotic to period doubling as shown in Fig.3(A), while decreasing $a_{1}=0.25$ change the behavior of the system from chaotic to limit cycle as shown in Fig.3(B). Further decreasing the value of $a_{1}=0.20$ change the dynamics of system (2) from chaotic to stable point $(0.472,0.714,0.816)$.

The second case about fixing the controlling parameter $a_{1}=0.35$ with same data in (23), and by increasing the value of $b_{1}$ about 2 -times to $b_{1}=0.6$ change 

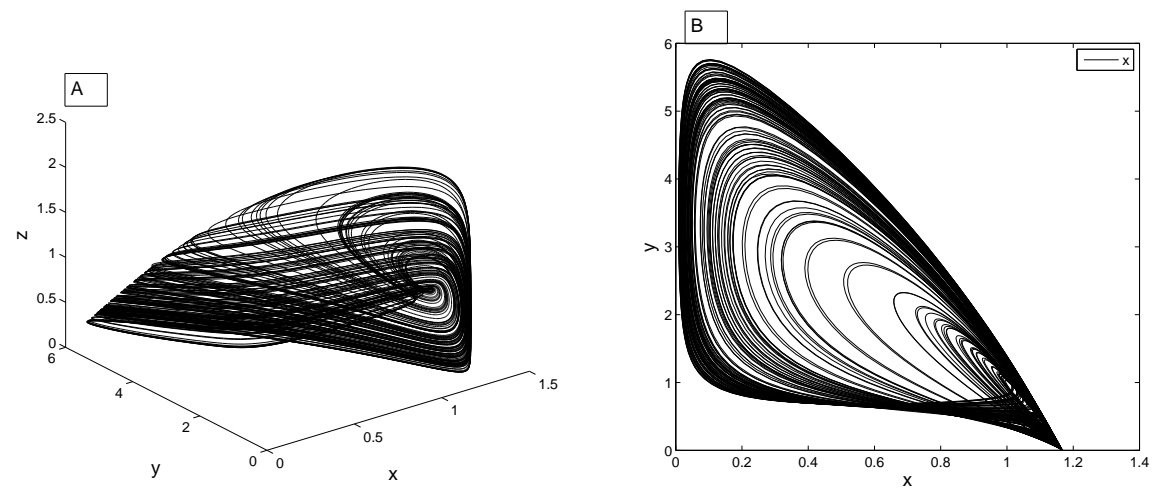

Figure 2: (A) 3D of system (2) chaotic attractor with data in (23) and $a_{1}=0.35$. (B) $2 \mathrm{D} x-y$ plane of Fig.2(A).
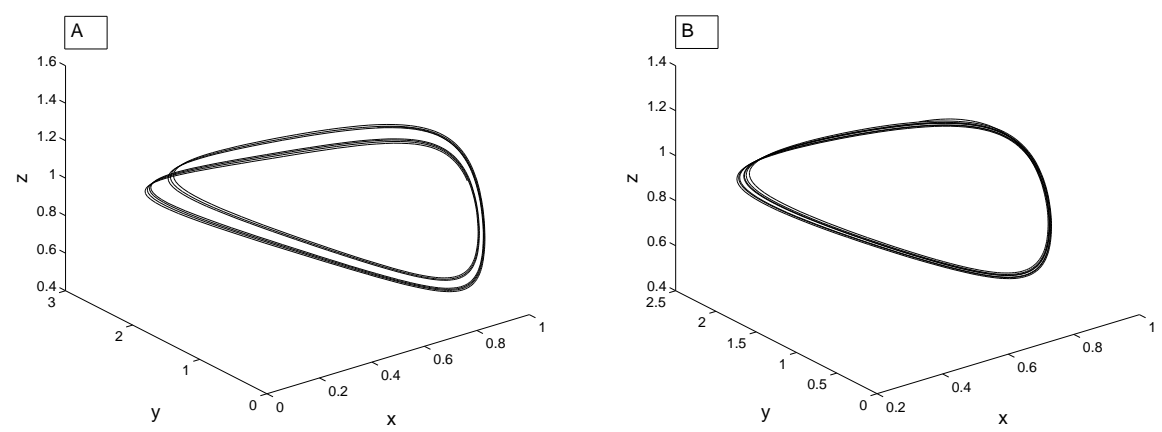

Figure 3: (A) 3D of system (2) period doubling attractor with data in (23) and $a_{1}=0.27$. (B) limit cycle attractor with data in (23) and $a_{1}=0.25$.

the behavior of system (2) dynamics from chaotic to limit cycle as shown in fig.4.(A), while increasing the value of $b_{1}$ about 4-times for the typical value of $b_{1}=1.2$ change the behavior of system (2) from chaotic to stable point $(0.235$, $0.710,0.402)$ as show in fig.4(B).

The third case about fixing $a_{1}=0.35$ with same data in (23), it is observed by increasing the death rate of the middle predator $d_{1}=0.034$ about 2 -times then system (2) approaches to periodic attractor as shown in Fig.5(A) and bifurcation diagram in Fig.6, while increasing the death rate of the top predator about 2-times $d_{2}=0.053$ change the behavior of system (2) from chaotic to 

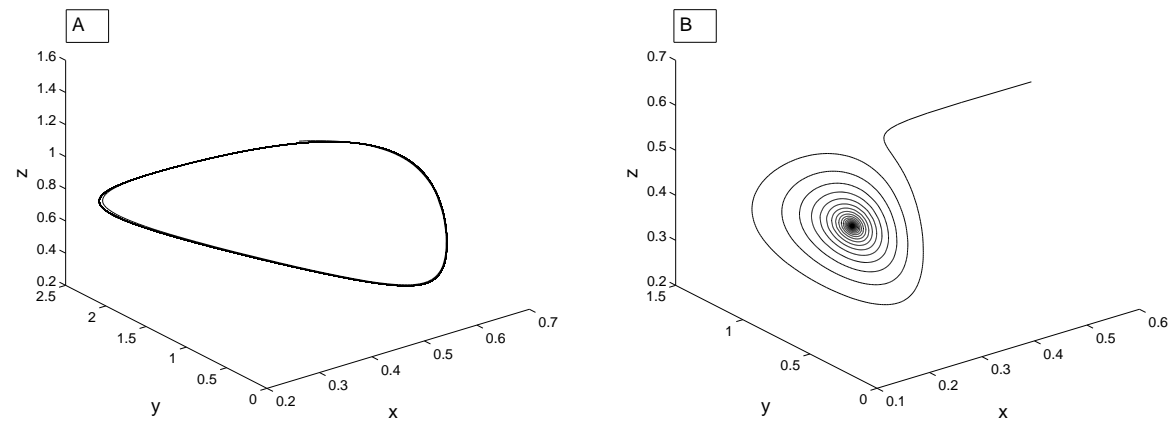

Figure 4: (A) 3D of system (2) periodic with data set (23) and $b_{1}=0.60$. (B) $3 \mathrm{D}$ of system (2) stable point with data set $(23)$ and $b_{1}=1.20$.
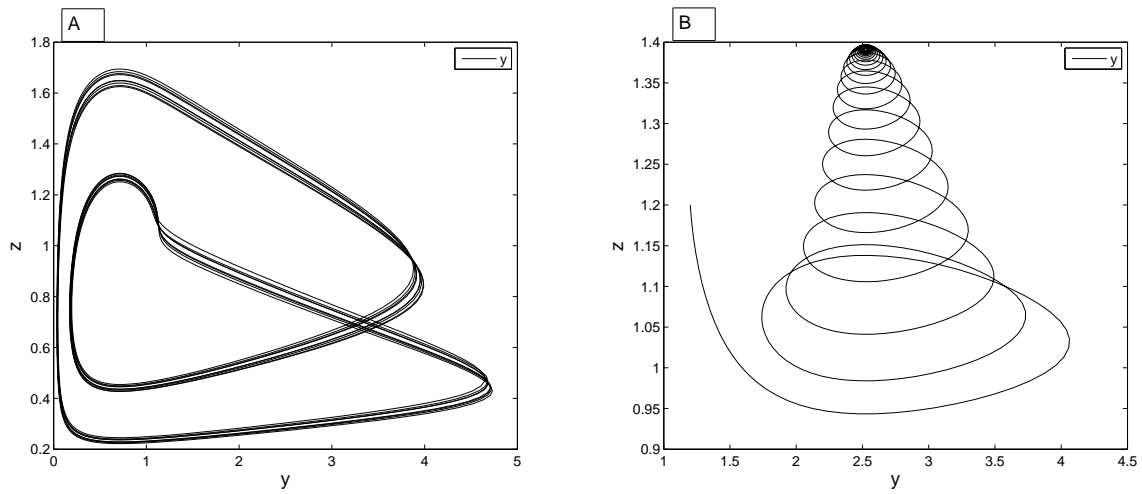

Figure 5: (A) 2D $y-z$ plane of system (2) period doubling with data in (23) and $d_{1}=0.034$. (B) $2 \mathrm{D} y-z$ plane of system (2) stable point with data in $(23)$ and $d_{2}=0.053$.

stable at the point $(0.482,2.524,1.392)$ as shown in Fig.5(B).

The bifurcation diagram with respect to $a_{1}$ with data in (23) is plotted in Fig.6 for $a_{1} \in(0.25,0.5)$ and $d_{1}=0.034$, the change in the dynamics of system (2) comparing with Fig. 1 when $d_{1}=0.017$ is observed. 


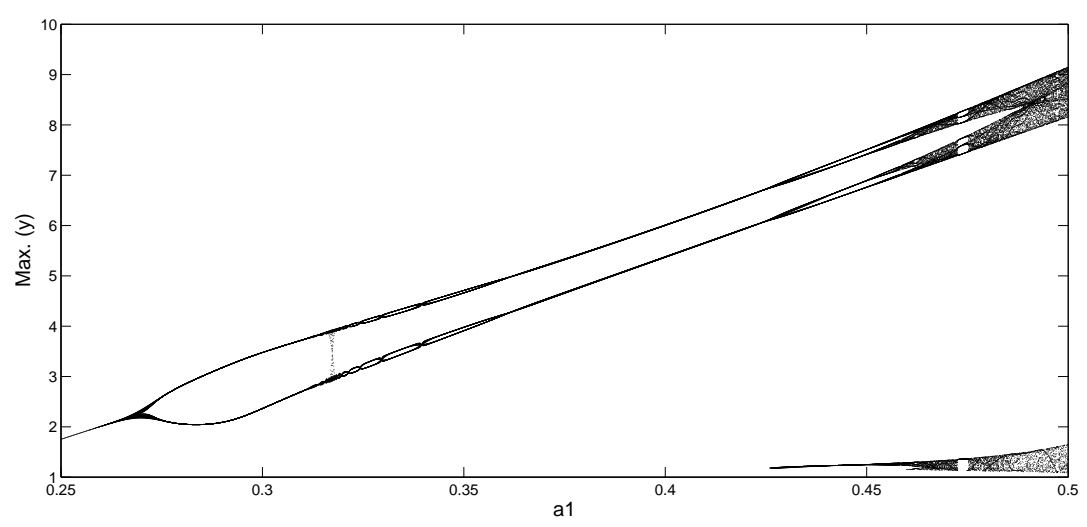

Figure 6: (A) Bifurcation diagram of system (2) for data in (23) the max. of $(y)$ as function of $a_{1}$ and $d_{1}=0.034$ with $a_{1} \in(0.25,0.5)$.

\section{Discussion and Results}

In order to explain the dynamical behavior of the proposed food chain systems (2), boundedness of the system is discussed. stability analysis of the model are carried out.

In addition to that, to confirm the analytical results the system is solved numerically for different sets of biologically feasible parameter values, and then the attracting sets are drown in order to explain the dynamical behavior of the model as in Figs.(1-6) with data the same as in (23). According to our study the following results are obtained:

1) The bifurcation diagrams indicate the existence of rich dynamics (chaotic, periodic, period doubling, asymptotically stable) for parameter range in a three species food chain model system (2).

2) System (2) has a chaotic dynamics as in Fig.2, but if we decreasing the values of intrinsic growth of the prey species $a_{1}$, then system (2) approaches to periodic attractor as in Fig.3, and then to stable point. So decreasing $a_{1}$ has a stabilizing effect on the dynamics of system (2).

3) Increasing the intraspecific competition change system (2) from chaotic to asymptotically stable, so increasing $b_{1}$ has a stabilizing effect on the dynamics of food chain system (2), see Fig.4.

4) Increasing the death rate $d_{1}$ in the middle predator $y$ about 2-times with 
data (23) change the dynamics of system (2) from chaotic to periodic as it was plotted in Fig.5(A). Moreover increasing the death rate $d_{2}$ in the top predator $z$ about 2-times change system (2) behavior from chaotic to stable as shown in fig.5(B), so $d_{2}$ is more effected than $d_{1}$ in the behavior of system (2).

\section{References}

[1] P. A. Abrams, Effect of increased productivity on the abundances of trophic level, American Naturalist, 141 (1993), 351-371.

[2] P. A. Abrams, J. D. Roth, The effects of enrichment of three species food chain with nonlinear functional response, Ecology, 75 (1994), 1118-1130.

[3] J. A. Andrews, The effects of enrichment of three species food chain with nonlinear functional response, Biotechnology and Bioengineering, 10(6) (1968), 707-723.

[4] M. A. Aziz-Alaoui, Study of a Leslie-Gower type tritrophic population model, Choas, Solitons and Fractals, 14 (2002), 1275-1293.

[5] M. P. Boer, B. W. Kooi and S. Kooijman, Multiple attractors and boundary crises in a tri-trophic food chain, Mathematical Biosciences, 169 (2001), 109-128.

[6] J. B. Collings, The effect of the functional response on the bifurcation behavior of a mite predator-prey interaction model, J. Math. Biol., 36 (1997), 149-168.

[7] O. De Feo and S. Rinaldi, Yield and dynamics of tritrophic food chain, American Naturalist, 150 (1997), 328-345.

[8] S. Gakkhar and R.K. Naji, Chaos in three-species ratio dependent food chain, Chaos,Solitons and Fractals, 14 (2002), 771-778.

[9] S. Gakkhar and A. Singh, Control and chaos du to additional predator in Hastings-powell food chain model, J. Math. Anal. appl., 385 (2012), 423-438.

[10] C. F. Gerald and PO. Wheatly, Applied numerical analysis, New York, USA, AdisonWesley, (2004).

[11] J.K. Hall, Ordinary differential equation First Edition. Wiley-Interscience, New-York, (1969).

[12] M. Haque, N. Ali and S. Chakravarty, Study of a tri-trophic prey-dependent food chain model of intracting populations, Mathematical Biosciences, 246 (2013), 55-71.

[13] A. Hasting and T. Powell, Chaos in three-species food chain, Ecology, 72 (1991), 896-903.

[14] C. S. Holling, The functional response of predators to prey density and its rule in mimicry and population regulation, Mem. Entomology. Soc. Can., 45 (1965), 3-60.

[15] A. Klebanoff and A. Hasting, Chaos in three species food chains, Journal of Mathematical Biology, 32 (5) (1994), 427-451.

[16] Ya. Kuznetsov and S. Rinaldi, Remarks on food chain dynamics, Math Biosci, 134 (1996), $1-33$.

[17] R.M. May, Stability and complexity in model ecosystems, Princeton University Press, Princeton, New Jersey, (1973). 
[18] R. K. Naji, R. K. Upadhyay and V. Rai, Dynamical consequences of predator interference in tri-trophic model food chain, Nonlinear Analysis:Real World Applications, 11 (2010), 809-818.

[19] V. Rai, R. Seernivasan, Period-doubling bifurcations leading to chaos in a model food chain, Ecological Modelling, 69(1) (1993), 63-77.

[20] R. K. Upadhyay, SRK. Iyengar, Introduction to mathematical modeling and chaotic dynamics, First Edition. CRC Press, A Chapman and Hall Book, (2014).

[21] C. Shen, Permanence and global attractivity of the food chain system with Holling type IV Functional response, Appl. Math. Comput., 215 (2009), 179-185.

[22] S. Ruan and D. Xiao, Global analysis in a predator-prey system with non-monotonic functional response, SIAM J. Appl. Math., 61 (4) (2001), 1445-1472.

[23] W. Sokol and J. A. Howell, The kinetics of phenol oxidation by washed cells, Biotechnology and Bioengineering, 30 (1987), 921-927. 
